\title{
Predicción del tipo de experiencia sexual en función de variables psicológicas y sociodemográficas en adolescentes panameños
}

\section{Predicting the kind of sexual experience in terms of sociodemographic and psychological variables in panamanians adolescents}

\author{
$\mathrm{M}^{\mathrm{a}}$ Teresa Ramiro \\ Ma Paz Bermúdez \\ Juan Carlos Sierra \\ Gualberto Buela-Casal \\ Universidad de Granada, España
}

(Rec: 02 de octubre de 2012 / Acept: 09 de noviembre de 2012)

\begin{abstract}
Resumen
El objetivo de este estudio es establecer un modelo predictivo del tipo de experiencia sexual, a través de la evaluación de una serie de variables psicológicas y sociodemográficas. La muestra, representativa de la población de adolescentes escolarizados de Chiriquí (Panamá), está formada por 1.897 adolescentes de ambos sexos, de edades comprendidas entre los 14 y los 18 años $(M=16.25 ; D T=1.47)$. Los resultados mostraron que existen diferencias significativas en las variables evaluadas en función del tipo de experiencia sexual. Además, el sexo, la religión, el nivel de estudios del padre, el locus de control interno, la autoeficacia, la comunicación con la madre, la comunicación con amigos y la susceptibilidad, resultaron ser variables predictoras del tipo de experiencia sexual.

Palabras clave: Experiencia sexual, Adolescentes, VIH, ETS
\end{abstract}

\begin{abstract}
The aim of this work is to determine whether sociodemographic and psychosocial variables predict the kind of sexual experience. Participants were 1.897 adolescents. Ages ranging from 14 to 18 years old $(M=16.25$; $S D=1.47$ ). The results showed significant differences in the variables evaluated according to the type of sexual experience. In addition, sex, religion, parent educational level, internal locus control, self-efficacy, communication with mother, communication with friends and susceptibility were found to be significant predictors of type of sexual experience.

Key words: sexual experience, adolescents, HIV, Sexually Transmitted Diseases.
\end{abstract}

\section{Introducción}

En algunos países más desarrollados se ha producido un descenso en el número de infectados por VIH (ONUSIDA, 2010; UNAIDS, 2010), sin embargo en América Latina no ha variado durante la última década. Concretamente, en Panamá, desde el año 1984 en el que se detecta el primer caso, hasta diciembre de 2009, se han contabilizado 10.381 casos de sida acumulados. Además, el grupo etáreo más afectado es el de 25 a 44 años de edad, y en el $69.1 \%$ de los casos la transmisión del VIH fue por vía sexual, siendo el 52.6\% a través de relaciones heterosexuales (Bermúdez y Teva, 2004). Asimismo, para el año 2009 se estimó una prevalencia del 0.9\% (Bermúdez y Teva, 2004).

Los adolescentes son un grupo de alta vulnerabilidad a la infección por el VIH, debido a variables de tipo biológico, socioeconómico y, principalmente, comportamental (Futterman, 2004; Paramo, 2011). En este sentido, diversos

Agradecimientos: Esta investigación ha sido realizada gracias a la subvención concedida por el Ministerio de Asuntos Exteriores y de Cooperación: Agencia Española de Cooperación Internacional para el Desarrollo (Referencia A/6666/06).

Correspondencia: M Teresa Ramiro, mail: sramiro@ugr.es Universidad de Granada. Facultad de Psicología, 18071 Granada (España) 
estudios han demostrado que el inicio temprano de las relaciones sexuales coitales es por sí misma una conducta de riesgo para las infecciones de transmisión sexual (Li et al., 2009; Mitchell, Whitsell, Spicer, Beals y Kaufman, 2007), ya que presentan un menor uso del preservativo y mayor número de parejas (Kalina et al., 2009; Zimmer-Gembeck y Helfand, 2008). Concretamente, en un estudio realizado con una amplia muestra de adolescentes de entre $14 \mathrm{y}$ 20 años, se concluye que cada año de retraso en el debut sexual aumenta en 1.44 veces la probabilidad de hacer uso del preservativo (Yotebieng, Halpern, Mitchell y Adimora, 2009). Por lo tanto, el perfil de riesgo de los adolescentes que inician su conducta sexual a una edad más temprana es significativamente más alto, aumentando la probabilidad de diagnóstico de una ITS y de embarazos no deseados (Dancy, Crittenden y Ning, 2010). El inicio sexual temprano ha sido relacionado con múltiples variables psicosociales, entre las cuales ciertas características sociodemográficas tienen un papel importante. Así, el inicio sexual ha sido relacionado con la estructura familiar, donde la convivencia en una unidad familiar intacta hace más probable que se retrase el debut sexual (Zimmer-Gembeck y Helfand, 2008), convirtiéndose en un factor de protección. Otros factores de protección son: el hecho de presentar un alto grado de religiosidad, pertenecer a un estrato socioeconómico más alto, tener unos padres con un mayor nivel de estudios y ser mujer, ya que los varones tienen mayor probabilidad de mantener relaciones sexuales más tempranas (Jordahl y Lohman, 2009; Koffi y Kawahara, 2008; Zimmer-Gembeck y Helfand, 2008).

En relación a las variables psicológicas, el conocimiento sobre aspectos relacionados con la transmisión de ITS/ VIH ha mostrado resultados inconsistentes, ya que se ha relacionado con un inicio sexual más temprano (Schmied y Reidl, 2008), mientras que otros estudios no (Mohtasham et al., 2009). Del mismo modo, investigaciones que han relacionado el inicio sexual con la comunicación con los padres sobre aspectos relacionados con la sexualidad, el embarazo y la transmisión de ITS/VIH ponen de manifiesto resultados contradictorios. Por un lado, diversos autores han encontrado que aquellos adolescentes que informan tener una mayor comunicación con sus padres muestran un comportamiento sexual más tardío, cuando el mensaje de los padres es negativo y de rechazo con respecto al sexo (Atienzo, Walter, Campero, Lamadrid-Figueroa y Gutiérrez, 2008; Bersamin et al., 2008; Dancy et al., 2010; Dilorio et al., 2006; Gilliam, Berlin, Kozloski, Hernández y Grundy, 2007; Lenciauskiene y Zaborkis, 2008), y por otro lado, existen estudios que muestran que altos niveles de comunicación con los padres están relacionados positivamente con las relaciones sexuales precoces (Bersamin et al., 2008). Otra variable comúnmente estudiada es la comunicación sobre aspectos relacionados con la práctica sexual, el embarazo y la transmisión de ITS/VIH con los amigos, ya que según algunos autores está directamente relacionada con un inicio sexual posterior (Busse, Fishbein, Bleakley y Hennessy, 2010). No obstante, según los mismos autores, dicha relación está mediada por la presión normativa del grupo de iguales (Gilliam et al., 2007; Potard, Coutois y Rusch, 2008; Zimmer-Gembeck y Helfand, 2008). Sin embargo, cabe destacar que se han encontrado diferencias de género, ya que para los varones la iniciación sexual es un elemento de socialización positiva dentro del grupo de amigos, mientras que para las mujeres es un componente más de una relación amorosa (Navarro-Pertusa, Reig-Ferrer, Heredia y Cascales, 2006).

Las actitudes negativas hacia cuestiones relacionadas con el sida, así como hacia las relaciones sexuales, han sido asociadas con el debut sexual más tardío en la adolescencia, mientras que actitudes más liberales y permisivas conllevan un inicio más precoz de la actividad sexual (Zimmer-Gembeck y Helfand, 2008). Sin embargo, a pesar de esta relación, también es necesario que los adolescentes presenten un mayor sentido de control personal sobre los comportamientos sexuales, mayor autoeficacia y mayor capacidad para rechazar mantener sexo, para poder permanecer inactivos sexualmente (Dancy et al., 2010; Gilliam et al., 2007; Mohtasham et al., 2009)

Por último, cabe destacar que los adolescentes que informan de su intención de no comenzar a mantener relaciones sexuales, presentan mayores niveles de susceptibilidad a contraer ITS/VIH (Makiwane y Mokomane, 2010; Mohtasham et al., 2009; Mwale, 2009), aunque los niveles de susceptibilidad son bajos especialmente entre los adolescentes varones (Vinaccia et al., 2007). Además, también es importante indicar que el factor psicológico locus de control para la salud puede explicar la emisión de conductas de riesgo, como el inicio sexual precoz (Alfaro, Torres, Soto y Díaz, 1998).

En función de la revisión bibliográfica realizada, el objetivo de este estudio es elaborar un modelo predictivo del tipo de experiencia sexual (no experimentados sexualmente, con experiencia sexual sin penetración y con experiencia sexual con penetración). Previamente, para justificar la introducción de una serie de variables psicosociales en el modelo predictor, se hallan las diferencias de éstas variables en función del tipo de experiencia sexual de los adolescentes. 


\section{Método}

\section{Diseño}

Se trata de un estudio ex post facto tipo prospectivo según la clasificación de Montero y León (Montero y León, 2007).

\section{Participantes}

La muestra está formada por 1.897 adolescentes de ambos sexos ( $46.6 \%$ varones y $53.4 \%$ mujeres), de edades comprendidas entre los 14 y los 18 años $(M=16.25 ; D T$ $=1.47$ ). De forma más detallada se pueden consultar las características sociodemográficas de la muestra en la Tabla 1.

\section{Instrumentos}

1. El Cuestionario de datos sociodemográficos. Este instrumento, elaborado Ad-hoc para la presente investigación, recoge datos sociodemográficos (edad, sexo, curso, lugar de residencia, nivel de estudio de los padres, situación laboral de los padres, estado civil de los padres, con quién vive, orientación religiosa, si tiene pareja y orientación sexual) y si ha tenido práctica sexual y de qué tipo (con penetración o no).

2. Escala VIH/SIDA-66. Versión reducida del cuestionario VIH/SIDA-164 (Bermúdez, Sánchez y Buela-Casal, 2003). Esta escala consta de cinco subescalas que son: a) Conocimiento sobre transmisión y prevención del VIH. Formada por 20 ítems en formato Verdadero, Falso y No sé, cuyo rango de puntuación oscila entre 0 y 20 . El alpha de Cronbach en el presente estudio para esta subescala fue de 0.65 . b) Ideas erróneas sobre transmisión y prevención del VIH, consta de 20 ítems en formato Verdadero, Falso y No sé, cuyo rango de puntuación también oscila entre 0 y 20 . El alpha de Cronbach en el presente estudio para esta subescala fue de 0.79 . c) Actitudes negativas hacia cuestiones relacionadas con el sida, consta de 12 ítems en formato Acuerdo/ Desacuerdo/No sé, cuyo rango de puntuación es de 0 a 12. El alpha de Cronbach en el presente estudio para esta subescala fue de 0.79. d) Susceptibilidad hacia la infección por VIH, que consta de 5 ítems en formato Acuerdo/Desacuerdo/No sé y cuya puntación máxima es 5 y su puntuación mínima 0 . El alpha de Cronbach en el presente estudio para esta subescala fue de 0.66. e) Autoeficacia, consta de 9 ítems en formato Acuerdo/Desacuerdo/No sé y tiene un rango de puntuación que oscila entre 0 y 9 . El alpha de Cronbach en el presente estudio para esta subescala fue de 0.76.
3. Cuestionario sobre comunicación parental (Wingood y Di Clemente, 1998). Mediante esta escala se obtiene información sobre la frecuencia de comunicación que el adolescente tiene con su padre y con su madre sobre sexualidad, uso de preservativos, ITS, sida y embarazos. Consta de 10 ítems con formato de respuesta tipo Likert de 4 puntos que van desde "Nunca" a "Frecuentemente". El rango de puntuación en ambas subescalas oscila entre 5 y 20 . El alpha de Cronbach en el presente estudio para los ítems de comunicación con madre fue de 0.83 y con padre de 0.85 .

4. Cuestionario sobre comunicación con amigos (Bermúdez, Sánchez y Buela-Casal, 2000a). Instrumento que recaba información sobre la frecuencia de comunicación que el adolescente tiene con sus amigos sobre sexo, uso de preservativos, ITS, sida y embarazos. Consta de 5 ítems con formato de respuesta tipo líkert de 4 puntos que van desde "Nunca" a "Frecuentemente", cuya máxima puntuación es 20 y la mínima 5 . El alpha de Cronbach en el presente estudio fue de 0.83 .

5. Escala sobre percepciones de normas del grupo de iguales (Bermúdez, Sánchez y Buela-Casal, 2000a). Está compuesta de 8 ítems, cuyo formato de respuesta es tipo Likert de 5 puntos que van desde "Totalmente en desacuerdo" a "Totalmente de acuerdo" y el rango de puntuación oscila entre 8 y 40 . El alpha de Cronbach en el presente estudio fue de 0.57 .

6. Escala sobre locus de control de salud adaptada para el SIDA (Bermúdez, Sánchez y Buela-Casal, 2000b). Consta de 9 ítems, 3 ítems de locus de control interno para la infección por el VIH con formato de respuesta tipo Likert de 5 puntos que van desde "Totalmente en desacuerdo" hasta "Totalmente de acuerdo" y el rango de puntuación oscila entre 3 y 15, y 6 ítems de locus de control externo, con el mismo formato de respuesta y un rango de puntuación de 6 a 30. El alpha de cronbach en el presente estudio fue de .70 para la subescala locus de control interno y de .69 para la subescala de locus de control externo.

\section{Procedimiento}

Se realizó un muestreo aleatorio y estratificado según el lugar de residencia y el curso, teniendo en cuenta los datos facilitados por el Ministerio de Educación, Dirección General de Chiriquí, sobre el número de adolescentes matriculados en centros de enseñanza de la Provincia de Chiriquí en cada distrito y cada curso. Para ello, se agruparon los distritos de la provincia de Chiriquí en tres grandes regiones: zona Occidental, zona Oriental y Tierras altas, de las cuales se 
seleccionaron colegios al azar. La muestra es representativa y se ajusta a la distribución según lugar de residencia y curso de la población general de estudiantes de secundaria de entre 14 y 18 años.

Todos los instrumentos de evaluación que se utilizaron fueron adaptados lingüísticamente a la población objeto de análisis. En el proceso de adaptación participaron, en una primera fase 12 jueces panameños expertos en la materia, y posteriormente 60 adolescentes residentes en Chiriquí.

Posteriormente, los investigadores, previamente entrenados en la aplicación de los cuestionarios, acudieron a los centros de enseñanza seleccionados donde la evaluación fue realizada en las aulas. Los cuestionarios se aplicaron, tras obtener el consentimiento informado, de forma colectiva en grupos de 15-20 alumnos durante 35 minutos aproximadamente, bajo las mismas condiciones para todos los participantes en un periodo de 2 meses. Se proporcionó a todos los estudiantes información sobre el estudio y se garantizó la confidencialidad y el anonimato de sus respuestas, así como que su participación era voluntaria.

\section{Resultados}

En primer lugar se calculó la asociación de las variables sociodemográficas estudiadas con la variable tipo de experiencia sexual, que se refiere al tipo de contacto sexual que ha mantenido el adolescente y consta de tres niveles: a) sin experiencia sexual (ninguna conducta de índole sexual); b) con experiencia sexual sin penetración (besos, caricias, masturbación); y c) con experiencia sexual con penetración (sexo vaginal y sexo anal). Los resultados encontrados pusieron de manifiesto que existen diferencias estadísticamente significativas según el sexo $\left(\chi_{(2)}^{2}=64.32 ; p=.000\right)$, el estado civil de los padres $\left(\chi_{(6)}^{2}=13.46 ; p=.036\right)$, el nivel de estudios del padre $\left(\chi_{(10)}^{2}=64.32 ; p=.000\right)$, el nivel de estudios de la madre $\left(\chi_{(10)}^{2}=36.41 ; p=.000\right)$, la religión $\left(\chi_{(4)}^{2}=19.31 ; p\right.$ $=.001)$, y el hecho de tener pareja estable $\left(\chi_{(2)}^{2}=155.76\right.$; $p=.000)$. En la Tabla 1 consultarse la distribución de los adolescentes en los diferentes niveles de cada variable en función de los 3 grupos. Por el contrario no se encontraron diferencias estadísticamente significativas en las otras variables sociodemográficas evaluadas.

Tabla 1. Datos sociodemográficos descriptivos en función de la variable tipo de experiencia sexual.

\begin{tabular}{|c|c|c|c|c|c|c|}
\hline & \multicolumn{2}{|c|}{$\begin{array}{l}\text { Sin experiencia sexual } \\
\qquad(\mathrm{N}=755)\end{array}$} & \multicolumn{2}{|c|}{$\begin{array}{c}\text { Con experiencia sexual SIN } \\
\text { penetración } \\
(\mathrm{N}=663)\end{array}$} & \multicolumn{2}{|c|}{$\begin{array}{c}\text { Con experiencia sexual CON } \\
\text { penetración } \\
(\mathrm{N}=479)\end{array}$} \\
\hline & Frecuencia & $\%$ & Frecuencia & $\%$ & Frecuencia & $\%$ \\
\hline \multicolumn{7}{|l|}{$\overline{\text { Sexo }}$} \\
\hline Varón & 277 & 36.7 & 320 & 48.3 & 286 & 59.9 \\
\hline Mujer & 478 & 63.3 & 343 & 51.7 & 197 & 40.1 \\
\hline$N$ & 755 & & 663 & & 479 & \\
\hline \multicolumn{7}{|l|}{$\overline{\text { Edad }}$} \\
\hline$M$ & 16.23 & & 16.28 & & 16.24 & \\
\hline DT & 1.61 & & 1.16 & & 1.63 & \\
\hline$N$ & 755 & & 663 & & 479 & \\
\hline \multicolumn{7}{|l|}{ Curso } \\
\hline Segundo & 33 & 4.4 & 19 & 2.9 & 50 & 10.4 \\
\hline Tercero & 89 & 11.8 & 69 & 10.4 & 45 & 9.4 \\
\hline Cuarto & 274 & 36.3 & 213 & 32.1 & 123 & 25.7 \\
\hline Quinto & 209 & 27.7 & 185 & 27.9 & 130 & 27.1 \\
\hline Sexto & 150 & 19.9 & 177 & 26.7 & 131 & 27.3 \\
\hline$N$ & 755 & & 663 & & 479 & \\
\hline \multicolumn{7}{|l|}{ Zona de residencia } \\
\hline Occidente & 314 & 41.6 & 281 & 42.4 & 236 & 49.3 \\
\hline Oriente & 229 & 30.3 & 202 & 30.5 & 141 & 29.4 \\
\hline Tierras altas & 212 & 28.1 & 180 & 27.1 & 102 & 21.3 \\
\hline$N$ & 755 & & 663 & & 479 & \\
\hline \multicolumn{7}{|l|}{ Religión } \\
\hline Católica & 416 & 56.3 & 403 & 61.6 & 312 & 66.8 \\
\hline Creyente de otra religión & 287 & 38.8 & 227 & 34.7 & 128 & 27.4 \\
\hline No creyente & 34 & 4.9 & 24 & 3.7 & 27 & 5.8 \\
\hline$N$ & 737 & & 654 & & 467 & \\
\hline
\end{tabular}




\begin{tabular}{|c|c|c|c|c|c|c|}
\hline & \multicolumn{2}{|c|}{$\begin{array}{l}\text { Sin experiencia sexual } \\
\qquad(N=755)\end{array}$} & \multicolumn{2}{|c|}{$\begin{array}{c}\text { Con experiencia sexual SIN } \\
\text { penetración } \\
(\mathrm{N}=663)\end{array}$} & \multicolumn{2}{|c|}{$\begin{array}{c}\text { Con experiencia sexual CON } \\
\text { penetración } \\
(\mathrm{N}=479)\end{array}$} \\
\hline & Frecuencia & $\%$ & Frecuencia & $\%$ & Frecuencia & $\%$ \\
\hline \multicolumn{7}{|l|}{ Con quién vive } \\
\hline Padres & 588 & 78.5 & 514 & 78.2 & 363 & 77.7 \\
\hline Familiares & 135 & 18.0 & 129 & 19.6 & 98 & 21.0 \\
\hline Internado & 26 & 3.5 & 14 & 2.1 & 6 & 1.3 \\
\hline$N$ & 749 & & 657 & & 467 & \\
\hline \multicolumn{7}{|l|}{ Estado civil de sus padres } \\
\hline Madre soltera & 86 & 11.5 & 85 & 13.1 & 61 & 12.9 \\
\hline Casados & 516 & 69.3 & 411 & 63.1 & 283 & 60.0 \\
\hline Separados & 122 & 16.4 & 134 & 20.6 & 109 & 23.1 \\
\hline Viudez de alguno de ellos & 21 & 2.8 & 21 & 3.2 & 19 & 4.0 \\
\hline$N$ & 745 & & 651 & & 472 & \\
\hline \multicolumn{7}{|l|}{ Nivel estudios madre } \\
\hline Sin escolaridad & 80 & 10.8 & 35 & 5.3 & 26 & 5.3 \\
\hline Primaria incompleta & 95 & 12.8 & 76 & 11.6 & 53 & 11.3 \\
\hline Primaria completa & 109 & 14.7 & 75 & 11.4 & 44 & 9.4 \\
\hline Secundaria incompleta & 138 & 18.6 & 145 & 22.0 & 111 & 23.8 \\
\hline Secundaria completa & 172 & 23.2 & 171 & 26.0 & 109 & 23.3 \\
\hline Universitarios & 146 & 19.7 & 156 & 23.7 & 124 & 26.6 \\
\hline$N$ & 740 & & 658 & & 467 & \\
\hline \multicolumn{7}{|l|}{ Nivel estudios padre } \\
\hline Sin escolaridad & 48 & 6.6 & 25 & 3.9 & 26 & 5.6 \\
\hline Primaria incompleta & 118 & 16.2 & 70 & 11.0 & 49 & 10.6 \\
\hline Primaria completa & 110 & 15.1 & 78 & 12.2 & 33 & 7.1 \\
\hline Secundaria incompleta & 126 & 17.3 & 138 & 21.6 & 100 & 21.6 \\
\hline Secundaria completa & 188 & 25.9 & 182 & 28.5 & 131 & 28.2 \\
\hline Universitarios & 137 & 18.8 & 145 & 22.7 & 125 & 26.9 \\
\hline$N$ & 727 & & 638 & & 464 & \\
\hline \multicolumn{7}{|l|}{ Situación laboral padres } \\
\hline Ambos trabajan fueran de casa & 255 & 34.2 & 252 & 38.6 & 212 & 44.8 \\
\hline Madre trabaja fuera de casa, padre no & 57 & 7.7 & 62 & 9.5 & 39 & 8.2 \\
\hline Padre trabaja fuera de casa, madre no & 335 & 45.0 & 278 & 42.6 & 185 & 39.1 \\
\hline Ninguno trabaja fuera de casa & 98 & 13.1 & 61 & 9.3 & 37 & 7.8 \\
\hline$N$ & 745 & & 653 & & 473 & \\
\hline \multicolumn{7}{|l|}{ Tiene pareja estable } \\
\hline$S i$ & 236 & 31.4 & 604 & 46.1 & 316 & 66.4 \\
\hline No & 515 & 68.6 & 656 & 53.9 & 160 & 33.6 \\
\hline$N$ & 751 & & 660 & & 476 & \\
\hline \multicolumn{7}{|l|}{ Orientación Sexual } \\
\hline Heterosexual & 695 & 92.1 & 623 & 96.1 & 437 & 94.8 \\
\hline Homosexual & 17 & 2.3 & 11 & 1.7 & 14 & 3.0 \\
\hline Bisexual & 14 & 1.9 & 14 & 2.2 & 10 & 2.2 \\
\hline$N^{\circ}$ & 726 & & 648 & & 461 & \\
\hline
\end{tabular}

Fuente. Elaboración de los autores a partir de los datos obtenidos a través de los análisis estadísticos realizados. 
En segundo lugar, se realizó un análisis de la covarianza múltiple para determinar la relación entre las variables psicológicas dependientes: conocimiento sobre transmisión y prevención del VIH, ideas erróneas sobre transmisión y prevención del VIH, actitudes negativas hacia cuestiones relacionadas con el sida, susceptibilidad hacia la infección por VIH, autoeficacia, comunicación parental sobre aspectos relacionados con el sexo, el VIH/ Sida y el embarazo, comunicación con amigos sobre aspectos relacionados con el sexo, el VIH/ Sida y el embarazo, percepción de normas del grupo de iguales y locus de control de salud, y la variable independiente "tipo de experiencia sexual", que tiene tres niveles: a) sin experiencia sexual, b) con experiencia sexual sin penetración, y, c) con experiencia sexual con penetración. Como puede observarse en la Tabla 2, los resultados mostraron que existen diferencias estadísticamente significativas entre los tres niveles del factor tipo de experiencia sexual en todas las variables psicológicas evaluadas, excepto en la variable locus de control externo para la infección por VIH $(\mathrm{F}=.99 ; p=.372)$. En la Tabla 3 , pueden observarse las puntuaciones medias obtenidas en cada una de las variables psicológicas evaluadas, en función de la variable tipo de experiencia sexual.

Debido a las diferencias encontradas entre los tres grupos, consecutivamente se realizó un análisis de regresión logística multinomial, con el objetivo de determinar qué variables independientes, de aquellas en las que los sujetos han mostrado diferencias, pueden predecir la variable dependiente tipo de experiencia sexual. Para ello se especificó un modelo de efectos principales de pasos sucesivos hacia delante, que

Tabla 2. Análisis de la covarianza múltiple de las variables psicológicas en función de la variable tipo experiencia sexual.

\begin{tabular}{|c|c|c|c|c|c|}
\hline & $\mathrm{SC}$ & $\mathrm{gl}$ & $\mathrm{MC}$ & $\mathrm{F}$ & $p$ \\
\hline 1. Conocimiento & 101.05 & 2 & 50.52 & 6.742 & .001 \\
\hline 2. Ideas erróneas & 109.47 & 2 & 54.73 & 5.098 & .006 \\
\hline 3. Actitud negativa & 93.32 & 2 & 46.66 & 5.864 & .003 \\
\hline 4. Susceptibilidad & 101.33 & 2 & 50.66 & 24.498 & .000 \\
\hline 5. Autoeficacia & 323.76 & 2 & 161.88 & 30.944 & .000 \\
\hline 6. Comunicación con padre & 265.46 & 2 & 132.74 & 7.487 & .001 \\
\hline 7. Comunicación con madre & 2.78 & 2 & 1.393 & .083 & .000 \\
\hline 8. Comunicación con amigos & 1398.69 & 2 & 699.34 & 44.736 & .000 \\
\hline 9. Percepción de normas del grupo de iguales & 1148.54 & 2 & 574.27 & 20.714 & .000 \\
\hline 10. Locus Control Interno & 232.912 & 2 & 116.45 & 10.523 & .000 \\
\hline 11. Locus Control Externo & 37.09 & 2 & 18.54 & .990 & .372 \\
\hline
\end{tabular}

Fuente. Elaboración de los autores a partir de los datos obtenidos a través de los análisis estadísticos realizados.

Nota. Abreviaturas. SC: Suma de cuadrados; gl: grados de libertad; MC: media cuadrática; F: estadístico F; $p$ : significación.

Tabla 3. Puntuaciones medias de las variables psicológicas en función de la variable tipo de experiencia sexual.

\begin{tabular}{|c|c|c|c|c|c|c|}
\hline & \multicolumn{2}{|c|}{ Sin experiencia sexual } & \multicolumn{2}{|c|}{$\begin{array}{l}\text { Experiencia sexual sin } \\
\text { penetración }\end{array}$} & \multicolumn{2}{|c|}{$\begin{array}{c}\text { Experiencia sexual con } \\
\text { penetración }\end{array}$} \\
\hline & $\mathrm{M}$ & DT & $\mathrm{M}$ & DT & $\mathrm{M}$ & DT \\
\hline 1. Conocimiento & 15.21 & 2.81 & 15.67 & 2.64 & 15.72 & 2.73 \\
\hline 2. Ideas erróneas & 3.88 & 3.49 & 3.31 & 3.10 & 3.60 & 3.29 \\
\hline 3. Actitud Negativa & 3.39 & 2.83 & 2.86 & 2.70 & 3.17 & 2.85 \\
\hline 4. Susceptibilidad & 3.11 & 1.52 & 3.63 & 1.35 & 3.53 & 1.43 \\
\hline 5. Autoeficacia & 5.58 & 2.41 & 6.15 & 2.23 & 6.64 & 2.22 \\
\hline 6. Comunicación con padre & 9.32 & 3.98 & 9.52 & 4.10 & 10.27 & 4.75 \\
\hline 7. Comunicación con madre & 12.24 & 4.11 & 12.15 & 3.98 & 12.14 & 4.26 \\
\hline 8. Comunicación con amigos & 12.57 & 4.11 & 13.99 & 3.91 & 14.86 & 3.70 \\
\hline 9. Percepción de normas del grupo de iguales & 25.50 & 5.33 & 27.10 & 5.26 & 27.26 & 5.28 \\
\hline 10. Locus Control Interno & 11.23 & 3.52 & 12.11 & 3.06 & 11.66 & 3.35 \\
\hline 11. Locus Control Externo & 11.46 & 4.47 & 11.32 & 4.22 & 11.69 & 4.27 \\
\hline
\end{tabular}

Fuente. Elaboración de los autores a partir de los datos obtenidos a través de los análisis estadísticos realizados.

Nota. Abreviaturas. M: puntuación media de los sujetos en la variable. DT: desviación típica 
Tabla 4. Resumen de los pasos para la inclusión de variables en el modelo.

\begin{tabular}{|c|c|c|c|c|c|}
\hline Modelo & Efecto & $-2 \log$ verosimilitud & $\mathrm{c}^{2}$ & $\mathrm{gl}$ & $p$ \\
\hline 0 & Intersección & 3560.82 & & & \\
\hline 1 & Sexo & 3284.11 & 56.08 & 2 & .000 \\
\hline 2 & Tener pareja & 3292.88 & 120.85 & 2 & .000 \\
\hline 3 & Comunicación con amigos & 3214.61 & 42.58 & 2 & .000 \\
\hline 4 & Susceptibilidad & 3195.62 & 23.59 & 2 & .000 \\
\hline 5 & Locus de control interno & 3182.90 & 10.88 & 2 & .004 \\
\hline 6 & Nivel estudios padre & 3194.65 & 22.62 & 10 & .012 \\
\hline 7 & Comunicación con Madre & 3181.87 & 9.84 & 2 & .007 \\
\hline 8 & Religión & 3182.39 & 10.37 & 4 & .035 \\
\hline 9 & Autoeficacia & 3178.27 & 6.25 & 2 & .044 \\
\hline
\end{tabular}

Fuente: Elaboración de los autores a partir de los datos obtenidos a través de los análisis estadísticos realizados.

Nota. Abreviaturas. c2: Prueba Chi-Cuadrado; gl: grados de libertad; p: significación.

Tabla 5. Número y porcentaje de casos clasificados correctamente por el modelo predictor del tipo de experiencia sexual.

\begin{tabular}{|c|c|c|c|c|}
\hline \multirow[b]{2}{*}{ Observado } & \multicolumn{3}{|c|}{ Pronosticado } & \multirow[b]{2}{*}{$\%$ correcto } \\
\hline & $\begin{array}{c}\text { Sin experiencia } \\
\text { sexual }\end{array}$ & $\begin{array}{c}\text { Con experiencia } \\
\text { sexual SIN } \\
\text { penetración }\end{array}$ & $\begin{array}{c}\text { Con experiencia } \\
\text { sexual CON } \\
\text { penetración }\end{array}$ & \\
\hline Sin experiencia sexual & 447 & 152 & 51 & 68.8 \\
\hline Con experiencia sexual SIN penetración & 214 & 281 & 91 & 48.0 \\
\hline Con experiencia sexual CON penetración & 112 & 165 & 132 & 23.3 \\
\hline$\%$ Total & 47.0 & 36.4 & 16.7 & 52.3 \\
\hline
\end{tabular}

Fuente. Elaboración de los autores a partir de los datos obtenidos a través de los análisis estadísticos realizados.

incluyera las siguientes variables independientes: el sexo, el estado civil de los padres, el nivel de estudios del padre y de la madre, la religión, el tener pareja, el conocimiento sobre transmisión y prevención del VIH, la susceptibilidad hacia la infección por VIH, la autoeficacia, la comunicación parental y la comunicación con amigos sobre aspectos relacionados con el sexo, el VIH/Sida y el embarazo, la percepción de normas del grupo de iguales y el locus de control interno de salud. Sin embargo, no se incluyeron las variables ideas erróneas sobre transmisión y prevención del VIH y actitudes negativas hacia cuestiones relacionadas con el sida, debido a que presentaban altas correlaciones con el resto de variables, haciendo que se incumpliese el supuesto de multicolinealidad.

En la Tabla 4 se puede observar el resumen de los pasos y las variables seleccionadas para la construcción del modelo con sus correspondientes valores de $\chi^{2}$, que indican la contribución individual de cada variable al ajuste global del modelo final $\left(\chi_{(28)}^{2}=379.80 ; p=.000\right)$. La prueba de bondad de ajuste del modelo resultó no ser significativa $\left(\chi^{2}{ }_{(210)}=198.70 ; p=.701\right)$, lo que indica que el modelo es adecuado para el ajuste de los datos, que también se comprobó con el estadístico $R^{2}$ de Nagelkerke, a través del cual se pudo determinar que la proporción de varianza explicada por el modelo obtenido es igual a 0.32. Además, como puede observarse en la Tabla 5, el modelo tiene una capacidad predictiva del $52.3 \%$ de los casos.

Por último, en la Tabla 6 se observan los resultados obtenidos acerca de las estimaciones de los parámetros de las variables incluidas en el modelo. Cabe destacar, que ser mujer reduce en un $23 \%$ la probabilidad de tener tanto relaciones sexuales sin penetración como con penetración. En relación al hecho de tener pareja estable, quienes no la tenían reducen la probabilidad de mantener relaciones sexuales sin penetración en un $37 \%$ y en un $22 \%$ la probabilidad de mantener relaciones sexuales con penetración, con respecto a quienes sí tenían pareja. Asimismo, aquellos adolescentes cuyo padre no había completado la primaria tuvieron un $30 \%$ menos de probabilidad de haber mantenido relaciones sexuales sin penetración y $57 \%$ menos de probabilidad de haber mantenido relaciones sexuales con penetración. Un factor de protección fue el pertenecer a una religión distinta 
de la católica, debido a que reduce en un $43 \%$ la probabilidad de mantener contactos sexuales coitales.

A cerca de las variables psicológicas incluidas en el modelo, los resultados mostraron que la probabilidad de mantener relaciones sexuales sin penetración aumenta 1.06 veces y con penetración 1.13 veces, a medida que aumenta en una unidad la comunicación con amigos. Además, la probabilidad de mantener relaciones sexuales coitales también aumenta 1.16 veces a medida que aumenta en una unidad la susceptibilidad, y la probabilidad de mantener relaciones sexuales sin penetración en 1.02 veces cuando el locus de control interno es mayor. Mayores puntuaciones en autoeficacia aumentan la probabilidad de mantener relaciones sexuales con penetración 1.07 veces, pero no aumenta la probabilidad de mantener relaciones sin penetración.

Por el contrario, la comunicación con la madre disminuye la probabilidad de mantener tanto relaciones sexuales sin penetración ( 0.96 veces) como relaciones sexuales con penetración (0.94 veces).

Tabla 6. Estimaciones de los parámetros de las variables incluidas en el modelo predictor del tipo de experiencia sexual.

\begin{tabular}{|c|c|c|c|c|c|c|c|c|}
\hline & $\mathrm{B}$ & E.T. & Wald & gl & $\mathrm{p}$ & OR & \multicolumn{2}{|c|}{ I.C. $95 \%$ OR } \\
\hline & & & & & & & Inferior & Superior \\
\hline \multicolumn{9}{|l|}{ Con experiencia sexual sin penetración } \\
\hline Sexo $=$ Varón & -0.749 & 0.477 & 2.767 & 1 & .096 & 0.452 & 0.1178 & 1.152 \\
\hline Sexo $=$ Mujer & -1.438 & 0.487 & 8.713 & 1 & .003 & 0.237 & 0.091 & 0.617 \\
\hline Pareja $=S i$ & $0^{\mathrm{b}}$ & . & . & 0 & . & . & . & . \\
\hline Pareja $=$ No & -0.976 & 0.126 & 60.247 & 1 & .000 & 0.377 & 0.295 & 0.482 \\
\hline Comunicación con amigos & 0.065 & 0.017 & 14.760 & 1 & .000 & 1.067 & 1.032 & 1.103 \\
\hline Susceptibilidad & 0.211 & 0.045 & 22.289 & 1 & .000 & 1.235 & 1.131 & 1.347 \\
\hline Locus de control interno & 0.058 & 0.020 & 8.699 & 1 & .003 & 1.060 & 1.020 & 1.101 \\
\hline Estudios padre $=$ sin escolarizar & -0.686 & 0.320 & 4.604 & 1 & .032 & 0.504 & 0.269 & 0.942 \\
\hline Estudios padre $=$ Primaria incompleta & -0.474 & 0.319 & 4.696 & 1 & .030 & 0.622 & 0.405 & 0.956 \\
\hline Estudios padre $=$ Primaria Completa & -0.353 & 0.214 & 2.737 & 1 & .098 & 0.702 & 0.462 & 1.067 \\
\hline Estudios padre $=$ Secundaria Incompleta & 0.031 & 0.194 & 0.026 & 1 & .872 & 1.032 & 0.706 & 1.509 \\
\hline Estudios padre $=$ Secundaria completa & -0.095 & 0.176 & 0.292 & 1 & .589 & 0.910 & 0.645 & 1.283 \\
\hline Estudios padre $=$ Universitarios & $0^{\mathrm{b}}$ & . & . & 0 & $\cdot$ & . & . & . \\
\hline Comunicación con madre & -0.036 & 0.016 & 5.003 & 1 & .025 & 0.964 & 0.934 & 0.996 \\
\hline Religión $=$ Católica & -0.019 & 0.322 & 0.004 & 1 & .952 & 0.981 & 0.522 & 1.845 \\
\hline Religión $=$ otra & -0.082 & 0.325 & 0.064 & 1 & .800 & 0.921 & 0.487 & 1.742 \\
\hline Religión $=$ No creyente & $0^{\mathrm{b}}$ & . & . & 0 & . & . & . & . \\
\hline Autoeficacia & 0.004 & 0.028 & 0.026 & 1 & .973 & 1.004 & 0.941 & 1.061 \\
\hline \multicolumn{9}{|l|}{ Con experiencia sexual con Penetración } \\
\hline Sexo $=$ Mujer & -1.461 & 0.537 & 7.391 & 1 & .007 & 0.232 & 0.081 & 0.665 \\
\hline Sexo $=$ Varón & -.407 & 0.523 & 0.606 & 1 & .346 & 0.666 & 0.239 & 1.854 \\
\hline Pareja $=$ Si & $0^{\mathrm{b}}$ & . & . & 0 & . & . & . & . \\
\hline Pareja $=$ No & -1.484 & 0.146 & 103.410 & 1 & .000 & 0.227 & 0.170 & 0.302 \\
\hline Comunicación con amigos & 0.127 & 0.020 & 39.858 & 1 & .000 & 1.136 & 1.092 & 1.182 \\
\hline Susceptibilidad & 0.152 & 0.051 & 8.765 & 1 & .003 & 1.164 & 1.053 & 1.287 \\
\hline Locus de control interno & 0.003 & 0.022 & 0.020 & 1 & .886 & 1.023 & 0.961 & 1.047 \\
\hline Estudios padre $=$ sin escolarizar & -0.368 & 0.327 & 1.265 & 1 & .261 & 0.692 & 0.365 & 1.314 \\
\hline Estudios padre $=$ Primaria incompleta & -0.561 & 0.252 & 4.966 & 1 & .026 & 0.571 & 0.348 & 0.935 \\
\hline Estudios padre $=$ Primaria completa & -0.938 & 0.271 & 12.027 & 1 & .001 & 0.391 & 0.230 & 0.665 \\
\hline Estudios padre $=$ Secundaria incompleta & -0.108 & 0.217 & 0.249 & 1 & .618 & 0.897 & 0.586 & 1.374 \\
\hline Estudios padre $=$ Secundaria completa & -0.190 & 0.197 & 0.936 & 1 & .333 & 0.827 & 0.562 & 1.216 \\
\hline Estudios padre $=$ Universitarios & $0^{\mathrm{b}}$ & . & . & 0 & 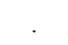 & . & . & 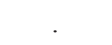 \\
\hline Comunicación con madre & -0.056 & 0.019 & 9.127 & 1 & .003 & 0.946 & 0.912 & 0.981 \\
\hline Religión $=$ Católica & -0.464 & 0.327 & 2.012 & 1 & .156 & 0.629 & 0.331 & 1.194 \\
\hline Religión $=$ otra & -0.832 & 0.335 & 6.155 & 1 & .013 & 0.435 & 0.226 & 0.840 \\
\hline Religión $=$ No creyente & $0^{\mathrm{b}}$ & . & . & 0 & . & . & . & . \\
\hline Autoeficacia & 0.076 & 0.033 & 5.191 & 1 & .023 & 1.079 & 1.011 & 1.152 \\
\hline
\end{tabular}

Fuente. Elaboración de los autores a partir de los datos obtenidos a través de los análisis estadísticos realizados.

Nota. Abreviaturas. B: coeficiente (B); E.T.: error típico de B; Wald: Estadístico de Wald; gl: grados de libertad; p; significación; OR: odds ratio 


\section{Discusión}

Los resultados que se derivan de este estudio son relevantes para tener en cuenta en la elaboración de estrategias preventivas, e indican que existen diferencias significativas entre los adolescentes en función del tipo de experiencia sexual. Dichos resultados permiten determinar, al igual que otras investigaciones (Jordahl y Lohman, 2009; Koffi y Kawahara, 2008; Zimmer-Gembeck y Helfand, 2008), que las variables sociodemográficas están fuertemente asociadas al debut sexual. No obstante, a pesar de que la mayoría de estudios determinan que la estructura familiar intacta, en la que no se ha producido separación de los cónyuges, es uno de los principales factores de protección (Zimmer-Gembeck y Helfand, 2008), y que en esta investigación se han encontrado diferencias estadísticas significativas, no ha resultado ser una variable predictora del inicio sexual. Por el contrario, en la misma línea de otros trabajos (Jordahl y Lohman, 2009; Koffi y Kawahara, 2008), el sexo, la religión y el nivel de estudios del padre han sido incluidas en el modelo predictor final, de tal modo que el hecho de ser mujer, pertenecer a una religión distinta de la católica, y un menor nivel de estudios del padre disminuyen la probabilidad de haber mantenido relaciones sexuales con penetración. Además, el hecho de no tener pareja estable, variable que no ha sido incluida en otros estudios, también es un claro predictor del retraso de comportamientos sexuales tanto con penetración como sin penetración. Por lo tanto, se trata de un aspecto novedoso que debería tenerse en cuenta en futuros programas de prevención destinados a promover la abstinencia.

En relación a las variables psicológicas, y tras demostrarse que los adolescentes presentaban diferencias estadísticamente significativas en casi todas las variables evaluadas, únicamente la comunicación con amigos y con la madre sobre aspectos relacionados con el sexo, el embarazo y la transmisión de ETS/VIH, la susceptibilidad hacia la infección por VIH, el locus de control interno para la infección por VIH y la autoeficacia, han resultado ser variables predictoras del tipo de experiencia sexual. La comunicación con la madre, ha mostrado ser un factor de protección ya que retrasa tanto el inicio de las relaciones sexuales con penetración como sin penetración. Estos resultados, que también han sido encontrados en otros estudios (Lenciauskiene y Zaborkis, 2008), indican la importancia de generar buenas pautas de interacción y comunicación entre padres e hijos, en oposición a otras investigaciones (Sánchez, Grogan-Kaylor, Castillo, Caballero y Delva, 2010), que indican que la relación que tienen los adolescentes con sus progenitores no influye en su comportamiento sexual. No obstante, la comunicación con el padre no solo no ha resultado ser una variable predictora, sino que se han obtenido diferencias estadísticas en sentido contrario, ya que son aquellos adolescentes que mantienen relaciones coitales los que más se comunican con su padre. Algunos autores indican que este fenómeno se produce porque, debido a los roles de género, la educación sexual dentro de la familia se delega en la mujer (Turnbull, Van-Wersch y Van-Scahaik, 2008). Por ello, es posible que el contenido de las conversaciones sea diferente en función de con cuál de los progenitores se mantengan. Así, la madre ejerce un papel educador mientras que el padre no, debido a que como ya se ha comentado anteriormente, el mensaje debe ser negativo y de rechazo respecto al sexo (Gilliam et al., 2007) para que produzca un retraso en el inicio sexual. Además, sería importante realizar análisis diferentes en función del sexo, debido a que es posible que hombres y mujeres se comuniquen con sus progenitores de forma desigual según sea con el padre o con la madre, así como estudiar el contenido de la comunicación, para determinar si se refuerza el comportamiento sexual o no.

Por el contrario, la comunicación con amigos sobre aspectos relacionados con el sexo, el embarazo y la transmisión de ITS/VIH ha mostrado ser un factor de riesgo, incrementando la probabilidad de mantener relaciones sexuales entre los adolescentes que presentan mayores tasas de comunicación con los amigos. Hay que tener en cuenta que a pesar de que la percepción de normas del grupo de iguales no ha resultado ser un predictor del tipo de experiencia sexual, sí se han encontrado diferencias significativas entre lo tres grupos, ya que aquellos que han mantenido relaciones sexuales tanto con penetración como sin penetración perciben mayores normas negativas con respecto a la conducta sexual segura por parte de su grupo de iguales. Por ello, como la comunicación con los amigos es una variable que se encuentra mediada por la normativa existente en el grupo de iguales (Gilliam et al., 2007; Potard et al., 2008; Zimmer-Gembeck y Helfand, 2008), se puede concluir, al igual que otros estudios, que altas tasas de comunicación con el grupo de iguales predice un debut sexual más temprano, si los adolescentes perciben una mayor permisividad sexual por parte de sus compañeros y una mayor proporción de compañeros que ya practican el coito (Potard et al., 2008; Shtarkshall, Carmel, Jaffe-Hirschfield y Wolosky-Wruble, 2009).

En relación a la susceptibilidad hacia la infección por $\mathrm{VIH}$, los resultados encontrados son contradictorios a otros estudios, que indican que mayores niveles de susceptibilidad retrasan el inicio sexual (Makiwane y Mokomane, 2010; Mohtasham et al., 2009; Mwale, 2009). No obstante, las 
mayores tasas de susceptibilidad encontradas en aquellos adolescentes que han mantenido relaciones sexuales tanto con penetración como sin penetración, pueden deberse a que han estado expuestos a conductas de riesgo, mientras que los adolescentes que no han mantenido ningún tipo de conducta sexual se sienten menos susceptibles porque no han tenido posibilidad de infección. Además, a pesar de que el conocimiento sobre transmisión y prevención del VIH no ha resultado significativo como predictor del tipo de experiencia sexual, sí ha presentado diferencias significativas entre los tres grupos, siendo los adolescentes que no han mantenido relaciones sexuales los que presentan menos conocimiento. Muchos estudios han demostrado la necesidad de tener un alto grado de conocimiento sobre aspectos relacionados con el sida para que se produzca susceptibilidad a la infección por VIH (Makiwane y Mokomane, 2010; Yotebieng et al., 2009). Por ello, también es posible que los adolescentes que no han mantenido relaciones sexuales presenten una menor susceptibilidad debido a que tienen menores tasas de conocimiento.

La variable autoeficacia ha resultado ser un factor de riesgo en contra de los datos obtenidos por anteriores estudios (Dancy et al., 2010; Gilliam et al., 2007; Mohtasham et al., 2009). Sin embargo, al igual que ocurre con la susceptibilidad, un explicación viable puede ser que, los adolescentes que han mantenido relaciones sexuales, presenten una mayor percepción de eficacia debido a que poseen mayor conocimiento y también mayor práctica en todos los aspectos relacionados con el comportamiento sexual.

Por último, cabe destacar que, en la misma línea que otras investigaciones (Alfaro et al., 1998), el locus de control interno para la infección por VIH ha sido significativo para predecir el comportamiento sexual sin penetración. Un alto nivel de locus de control interno permite que los adolescentes puedan desarrollar mejores habilidades conductuales, por ello, además de fomentar la abstinencia también es importante para generar conductas sexuales seguras.

La promoción de la abstinencia en los adolescentes ha sido cuestionada por algunos autores, debido a que piensan que puede ser equívoco para los propios jóvenes, por lo que los esfuerzos destinados a la prevención del VIH entre adolescentes, generalmente han sido destinados a promover conductas sexuales seguras, tales como el uso consistente del preservativo (Piña, Robles y Rivera, 2007). No obstante, un estudio reciente ha determinado que las consecuencias derivadas de los programas destinados a promover la abstinencia en diversos países, reducen los comportamientos de riesgo a corto y a largo plazo (Underhill, Operario y Montgomery, 2007). En este sentido, se ha constatado que el uso del preservativo en el primer contacto coital, cuya probabilidad es mayor a medida que se retrasa el inicio sexual, aumenta la probabilidad de usar preservativo en las relaciones sexuales más recientes en 4.81 veces, y el uso consistente en todas las relaciones en 8.76 veces (Yotebieng et al., 2009). Por todo ello, de este estudio se desprende la necesidad de intervenir preventivamente a edades tempranas promoviendo el retraso del inicio sexual, para lo que se deberá actuar fomentando la comunicación entre los adolescentes y sus padres, a la vez que se interviene sobre éstos para que puedan transmitir la necesidad de llevar a cabo estrategias preventivas eficaces en su comportamiento sexual, y desarrollando habilidades de resistencia hacia la presión normativa del grupo de iguales. Del mismo modo, también se debe incluir en los programas preventivos actividades que aumenten el conocimiento sobre la transmisión sobre ITS/VIH, la autoeficacia, la susceptibilidad hacia la infección y el locus de control interno para generar conductas sexuales seguras en el momento en el que se inicie el comportamiento sexual (Gutiérrez-Martínez, Bermúdez, Teva y Buela-Casal, 2007; Winskell, Obyerodhyambo y Stepheson, 2011).

Entre las principales limitaciones del estudio, se encuentra el hecho de no ser un estudio longitudinal, que permitiese poder hacer predicciones más efectivas. Por ello, en futuras investigaciones, se plantea la necesidad de realizar estudios de tipo longitudinal, y además, realizar modelos predictivos diferentes en función del sexo, debido a que se ha determinado que los varones se inician más temprano que las mujeres, y por ello, puede que las variables predictoras actúen de forma desigual. Otro aspecto a considerar, es la necesidad de llevar estudios con población adolescente panameña que permitan determinar el efecto del inicio sexual temprano sobre las conductas sexuales de riesgo. Además, un reciente estudio (Bermúdez, Castro, Madrid y Buela-Casal, 2010) ha puesto de manifiesto que los adolescentes de origen latinoamericano residentes en España se encuentran en mayor riesgo que los adolescentes autóctonos, sin embargo no se discrimina en función del país de origen. Por ello, se considera conveniente realizar estudios similares transculturales en diferentes países debido a que las variables estudiadas se encuentran íntimamente relacionadas con las distintas prácticas culturales (Bermúdez et al., 2010). 


\section{Referencias}

Alfaro, B., Torres, K. B., Soto, C., y Díaz, R. (1998). Factores sociodemográficos relacionados con el Locus de Control ante el VIH/SIDA en adolescentes. México D.F.: Congreso Nacional de Psicología Social.

Atienzo, E. E., Walter, D. M., Campero. L., Lamadrid-Figueroa, H., y Gutierrez, J.P. (2009). Parent-adolescent communication about sex in Morelos, Mexico: does it impact sexual behaviour? The European Journal of Contraception and Reproductive Health, 14, 111-119.

Bermúdez, M. P., Castro, A., Madrid, J., y Buela-Casal, G. (2010). Análisis de la conducta sexual de adolescentes autóctonos e inmigrantes latinoamericanos en España. International Journal of Clinical and Health Psychology, 10, 89-103

Bermúdez, M. P., Sánchez, A. I., y Buela-Casal, G. (2000a). Adaptación castellana de la escala sobre percepciones de normas del grupo de iguales. Avances en Psicología Latinoamericana, $18,57-70$

Bermúdez, M. P., Sánchez, A. I., y Buela-Casal, G. (2000b). Adaptación castellana de la escala sobre locus de control de salud modificada para el SIDA. Revista Mexicana de Psicología, 1, 27-35.

Bermúdez, M. P., Sánchez, A. I., y Buela-Casal, G. (2003). Adaptación castellana del cuestionario VIH/SIDA-164. Revista Mexicana de Psicología, 20, 95-112.

Bermúdez, M. P., y Teva, I. (2004). Situación actual del VIH/SIDA en Europa: análisis de las diferencias entre países. International Journal of Clinical and Health Psychology, 3, 89-106.

Bersamin, M., Told, M., Fisher, D.A., Hill, D. L., Grube, J. W., y Walker, S. (2008). Parenting practices and adolescent sexual behavior: A longitudinal study. Journal of Marriage and Family, 70, 97-112.

Busse, P., Fishbein, M., Bleakley, A., y Hennessy, M. (2010). The Role of Communication With Friends in Sexual Initiation. Communication Research, 37, 239-255.

Dancy, B. L., Crittenden, K. S., y Ning, H. Y. (2010). African-American adolescents girls initiation of sexual activity. Women's Health Issues, 20, 146-155.

Dilorio, C., Resnicow, K., McCarty, F., De, A.K., Dudley, W. N., ...Wang, D.T.(2006). Keepin' it REAL! Results of a mother-adolescent HIV prevention. Western Journal of Nursing Research, 55, 43-51.

Futterman, D. (2004). HIV and AIDS in adolescents. Adolescent Medicine Clinics, 15, 369-382.

Gilliam, Gl., Berlin, A., Kozloski, M., Hernandez, M., y Grundy, M. (2007). Interpersonal and personal factors influencing sexual debut among Mexican-American young women in the United States. Journal of Adolescent Health, 41, 495-503.

Gutiérrez-Martínez, O., Bermúdez, M. P., Teva, I., y Buela-Casal, G. (2007). Sexual sensation-seeking and worry about sexually transmitted diseases (STD) and human immunodeficiency virus (HIV) infection among Spanish adolescents. Psicothema, 19, 661-666.

Jordahl, T., y Lohman, B.J. (2009). A bioecological analysis of risk and protective factors associated with early sexual intercourse of young adolescents. Children and Youth Services Review, 31, 1272-1282.

Kalina, O., Geckova, A. M., Jarkuska, P., Orosova, O., Van Dijk, J. P., y Reijneveld, S. A. (2009). Psychological and behavioural factors associated with sexual risk behaviour among Slovak students. BMC Public Health, 9 (15). Recuperado desde http://www.biomedcentral. com/1471-2458/9/15.
Koffi, A. K., y Kawahara, K. (2008). Sexual abstinence behavior among never-married youths in a generalized HIV epidemic country: evidence from the 2005 Cote d'Ivoire AIDS indicator surveyce. BMC Public Health, 8 (408). Recuperado desde http://www.ncbi.nlm.nih. gov/pmc/articles/PMC2628901/

Lenciauskiene, I., y Zaborkis, A. (2008). The effects of family structure, parent-child relationship and parental monitoring on early sexual behaviour among adolescents in nine European countries. Scandinavian Journal of Public Health, 36, 607-618.

Li, S. H., Huang, H., Cai, Y., Xu, G., Huang, F. R., y Shen, X. M. (2009). Characteristics and determinants of sexual behavior among adolescents of migrant workers in Shangai (China). BMC Public Health, 9 (195). Recuperado desde http://www. biomedcentral.com/1471-2458/9/195.

Ma, Q. Q., Ono-Kihara, M., Cong, L. M., Xu, G. Z., Pan, X. H., ...Zamani, S. (2009) Early initiation of sexual activity: risk factor for sexuallity transmitted diseases, HIV infection, and and unwanted pregnancy among university students in China. BMC Public Health, 9 (111). Recuperado desde http://www. ncbi.nlm.nih.gov/pmc/articles/PMC2674603/

Makiwane, M., y Mokomane, Z. (2010). South Africa youths' higher-risk sexual behaviour: an eco-developmental analysis. African Journal of AIDS Research, 9, 17-24.

Mitchell, C. M., Whitesell, N. R., Spicer, P., Beals, J., y Kaufman, C. E. (2007). Cumulative risk for early sexual initiation among American Indian youth: A discrete-time survival analysis. Journal of Research on Adolescence, 17, 387-411.

Mohtasham, G., Shamsaddin, N., Bazargan, M., Anosheravan, K., Elaheh, M., y Fazlolah, G. (2009). Correlates of the Intention to Remain Sexually Inactive Among Male Adolescents in an Islamic Country: Case of the Republic of Iran. Journal of School Health, 79, 23-129.

Montero, I., y León, O. G. (2007). A guide for naming research studies in Psychology. International Journal of Clinical and Health Psychology, 7, 847-862.

Mwale, M. (2009). Behavioural change vis-a-vis HIV\&AIDS knowledge mismatch among adolescents. The case of some selected schools in Zomba, Malawi. South African Journal of Science, 39, 460-467.

Navarro-Pertusa, E., Reig-Ferrer, A., Heredia, E. B., y Cascales, R. I. F. (2006). Peer group and adolescent sexual debut: Gender differences. International Journal of Clinical and Health Psychology, 6, 79-96.

ONUSIDA. Informe Nacional sobre los progresos realizados en la aplicación del UNGASS 2010 en Panamá. Ginebra: ONUSIDA; 2010. Recuperado desde http://data.unaids.org/pub/ Report/2010/panama_2010_country_progress_report_es.pdf

Páramo, M. A. (2011). Factores de riesgo y factores de protección en la adolescencia: Análisis de contenido a través de grupos de discusión. Terapia Psicológica, 29, 85-95.

Piña, J. A., Robles, R., y Rivera, B. M. (2007). Instrumento para la evaluación de variables psicológicas y comportamientos sexuales de riesgo en jóvenes de dos centros universitarios de México. Revista Panamericana de Salud Pública, 22, 295-303.

Potard, C., Courtois, R., y Rusch, E. (2008). The influence of peers on risky sexual behaviour during adolescence Source. European Journal of Contraception and Reproductive Health Care, 13, 264-270.

Sánchez, N., Grogan-Kaylor, A., Castillo, M., Caballero, G., y Delva, J. (2010). Sexual intercourse among adolescents in 
Santiago, Chile: a study of individual and parenting factors. Revista Panamericana de Salud Pública, 28, 267-274.

Schmied, G., y Reidl, C. (2008). Gender Roles, Sexuality and Sexual Education from the Perspective of Male Adolescents. Schweizerische Rundschau fur Medizin Praxis, 48, 319-341.

Shtarkshall, R. A., Carmel, S., Jaffe-Hirschfield, D., y WoloskiWruble, A. (2009). Sexual Milestones and Factors Associated with Coitus Initiation Among Israeli High School Students. Archives of Sexual Behavior, 38, 591-604.

Turnbull, T., Van Wersch, A., y Van Schaik, P. (2008). A review of parental involvement in sex education: The role for effective communication in British families. Health Education Journal, 67, 182-195.

UNAIDS. Global report: UNAIDS report on the global AIDS epidemic 2010. Ginebra: UNAIDS; 2010. Recuperado desde http:// www.unaids.org/documents/20101123_GlobalReport_em.pdf.

Underhill, K., Operario, D., y Montgomery, P. (2007). Systematic review of abstinence-plus HIV prevention programs in highincome countries. PLoS Medicine, 4, 1471-1485.

Vinaccia, S., Quiceno, J. M., Gaviria, A. M., Soto, A. M., GilLlario, M. D., y Ballester-Arnal, R. (2007). Conductas sexuales de riesgo para la infección por Vih/Sida en Adolescentes Colombianos. Terapia Psicológica, 25, 39-50.

Wingood, G. M., y DiClemente, R. (1998). Partner influences and gender-related factors associated with noncondom use among young adult African American women. American Journal of Community Psychology, 26, 29-51.

Winskell, K., Obyerodhyambo, O., y Stephenson, R. (2011). Making sense of condoms: Social representations in young people's HIV-related narratives from six African countries. Social Science \& Medicine, 72, 953-961.

Yotebieng, M., Halpern, C. T., Mitchell, E. M. H., y Adimora, A. A. (2009). Correlates of condom use among sexually experienced secondary school male students in Nairobi, Kenya. Journal of Social Aspects of HIV/AIDS SAHARA-J, 6, 9-16.

Zimmer-Gembeck, M. J., y Helfand, M. (2008). Ten years of longitudinal research on US adolescent sexual behavior: Developmental correlates of sexual intercourse, and the importance of age, gender and ethnic background. Developmental Review, $28,153-224$ 